A N N A LES

UNIVERSITATIS MARIAE CURIE-SKŁODOWSKA

LUBLIN - POLONIA

ANDRZEJ MICHALSKI

\title{
Sufficient conditions for quasiconformality of harmonic mappings of the upper halfplane onto itself
}

\begin{abstract}
In this paper we introduce a class of increasing homeomorphic self-mappings of $\mathbb{R}$. We define a harmonic extension of such functions to the upper halfplane by means of the Poisson integral. Our main results give some sufficient conditions for quasiconformality of the extension.
\end{abstract}

1. Introduction. Let $F$ be a complex-valued sense-preserving diffeomorphism of the upper halfplane $\mathbb{C}^{+}:=\{z \in \mathbb{C}: \operatorname{Im} z>0\}$ onto itself, where $\mathbb{C}$ stands for the complex plane. Then the Jacobian

$$
\mathrm{J}_{F}:=|\partial F|^{2}-|\bar{\partial} F|^{2}
$$

is positive on $\mathbb{C}^{+}$and so the function

$$
\mathbb{C}^{+} \ni z \mapsto \mathrm{D}_{F}(z):=\frac{|\partial F(z)|+|\bar{\partial} F(z)|}{|\partial F(z)|-|\bar{\partial} F(z)|}
$$

is well defined. We recall that $\mathrm{D}_{F}(z)$ is called the maximal dilatation of $F$ at $z \in \mathbb{C}^{+}$. Here and in the sequel $\partial:=\left(\partial_{x}-i \partial_{y}\right) / 2$ and $\bar{\partial}:=\left(\partial_{x}+i \partial_{y}\right) / 2$ stands for the formal derivatives operators. From the analytical characterization of quasiconformal mappings (see [3]) it follows that for any $K \geq 1, F$ is

2000 Mathematics Subject Classification. 30C55, 30C62.

Key words and phrases. Harmonic mappings, Poisson integral, quasiconformal mappings. 
$K$-quasiconformal if and only if

$$
\mathrm{D}_{F}(z) \leq K, \quad z \in \mathbb{C}^{+} .
$$

Assume now that $\mathrm{F}$ is quasiconformal, i.e. $F$ satisfies (1.3) for some $K \geq 1$. Then $F$ has a unique homeomorphic extension $F^{*}$ to the closure $\overline{\mathbb{C}^{+}}:=$ $\mathbb{C}^{+} \cup \hat{\mathbb{R}}, \hat{\mathbb{R}}:=\mathbb{R} \cup\{\infty\}$ (see [3]). The famous result of Beurling and Ahlfors (see [1]) says that a function $f$ of $\mathbb{R}$ onto itself is the restriction of $F^{*}$ if and only if $f$ is quasisymmetric, i.e. $f$ is a strictly increasing homeomorphism, such that

$$
\frac{1}{M} \leq \frac{f(x+t)-f(x)}{f(x)-f(x-t)} \leq M
$$

for some constant $M \geq 1$ and for all $x \in \mathbb{R}$ and $t>0$.

Assume additionally that $F$ is a harmonic mapping, i.e. $F$ satisfies the Laplace equation $\partial \bar{\partial} F=0$ on $\mathbb{C}^{+}$. Kalaj and Pavlović proved in [2] that an increasing homeomorphism $f$ of $\mathbb{R}$ onto itself is the restriction of $F^{*}$ if and only if it is biLipschitz and the Hilbert transformation of $f^{\prime}$ is bounded.

Following the idea of Beurling and Ahlfors we are going to find an effective extension of $f$ to $F^{*}$. For $f \in \mathcal{F}$, where $\mathcal{F}$ is considered in Section 2, we provide a construction of the harmonic extension $H[f]$ defined in Definition 3.1 by means of the Poisson integral. The main purpose of this paper is to give sufficient conditions on $f \in \mathcal{F}$, that guarantee quasiconformality of $H[f]$. In Section 3 we show that $H[f]$ is a homeomorphism of $\mathbb{C}^{+}$onto itself provided $f \in \mathcal{F}$ has the biLipschitz property (3.2), cf. Proposition 3.2. In Section 4 we provide various auxiliary estimates dealing with partial derivatives of $H[f]$. Applying them we are able to estimate the maximal dilatation $\mathrm{D}_{H[f]}$ of $H[f]$ in case $f \in \mathcal{F}$ satisfies the biLipschitz property (3.2) and $f^{\prime}$ is a Dini-continuous function with respect to spherical distance (4.3). This is the main result of the paper and is stated in Theorem 5.2. In particular, if $f^{\prime}$ is Hölder-continuous with respect to spherical distance we obtain estimate of $\mathrm{D}_{H[f]}$ given in Theorem 5.3.

2. Preliminary notes. Let $\mathrm{Hom}^{+}(\mathbb{R})$ be the set of all increasing real line homeomorphisms onto itself. For $a \in \mathbb{R}$ we define

$$
\mathcal{F}_{a}:=\left\{f \in \operatorname{Hom}^{+}(\mathbb{R}): \mathrm{I}(f, a)<+\infty\right\},
$$

where

$$
\mathrm{I}(f, a):=\int_{-\infty}^{+\infty} \frac{|f(t)-a t|}{1+t^{2}} \mathrm{~d} t
$$

We define also

$$
\mathcal{F}:=\bigcup_{a>0} \mathcal{F}_{a} .
$$

The following properties hold. 
Proposition 2.1. If $a<0$, then $\mathcal{F}_{a}=\emptyset$.

Proof. Let $f \in \operatorname{Hom}^{+}(\mathbb{R})$. There exists $T>0$ such that $f(t) \geq 0$ for $t \geq T$. Hence, if $a<0$, then $|f(t)-a t| \geq f(t)+|a| t$ for $t \geq T$, which implies that

$$
\mathrm{I}(f, a) \geq \int_{T}^{+\infty} \frac{f(t)+|a| t}{1+t^{2}} \mathrm{~d} t .
$$

Since the last integral is divergent, $f \notin \mathcal{F}_{a}$ and we have a contradiction which completes the proof.

Proposition 2.2. If $a \neq b$, then $\mathcal{F}_{a} \cap \mathcal{F}_{b}=\emptyset$.

Proof. Let $f \in \mathcal{F}_{a} \cap \mathcal{F}_{b}, a \neq b$. Observe, that

$$
\int_{-\infty}^{+\infty} \frac{|(a-b) t|}{1+t^{2}} \mathrm{~d} t \leq \int_{-\infty}^{+\infty} \frac{|f(t)-a t|}{1+t^{2}} \mathrm{~d} t+\int_{-\infty}^{+\infty} \frac{|f(t)-b t|}{1+t^{2}} \mathrm{~d} t<+\infty .
$$

But the first integral is divergent, thus we have a contradiction, which completes the proof.

Remark 2.3. By Proposition 2.2, for every fixed $f \in \mathcal{F}$ there exists exactly one constant $a>0$, such that $\mathrm{I}(f, a)<+\infty$.

Proposition 2.4. If $f \in \mathcal{F}_{a}$, then $\tilde{f} \in \mathcal{F}_{a}$, where $\tilde{f}(t):=-f(-t), t \in \mathbb{R}$.

Proof. Consider I $(\tilde{f}, a)$. Substituting $s:=-t$ we have

$$
\mathrm{I}(\tilde{f}, a)=-\int_{+\infty}^{-\infty} \frac{|\tilde{f}(-s)+a s|}{1+s^{2}} \mathrm{~d} s=\int_{-\infty}^{+\infty} \frac{|-\tilde{f}(-s)-a s|}{1+s^{2}} \mathrm{~d} s=\mathrm{I}(f, a) .
$$

Proposition 2.5. If $f \in \mathcal{F}$, then $\liminf _{t \rightarrow+\infty} f(t) / t \geq 0$.

Proof. Assume that $\liminf \operatorname{in}_{t \rightarrow+\infty} f(t) / t<0$, then there exists a sequence $\left\{t_{n}\right\}$ and $T \in \mathbb{R}$ such that $t_{n} \rightarrow+\infty$ and $f\left(t_{n}\right)<0$ for $n \geq T$. But $f \in \operatorname{Hom}^{+}(\mathbb{R})$, i.e. $f$ is an increasing homeomorphism of $\mathbb{R}$ onto $\mathbb{R}$, thus we have a contradiction and the proof is completed.

Proposition 2.6. If $f \in \mathcal{F}$, then $\liminf _{t \rightarrow-\infty} f(t) / t \geq 0$.

Proof. Consider $\tilde{f}(t):=-f(-t)$. By Proposition 2.4 we have $\tilde{f} \in \mathcal{F}_{a}$ and then by Proposition 2.5 we have

$$
\liminf _{t \rightarrow+\infty} \tilde{f}(t) / t \geq 0
$$

This is equivalent to $\liminf \operatorname{int}_{t \rightarrow-\infty} f(t) / t \geq 0$, which completes the proof.

Proposition 2.7. If $f \in \mathcal{F}_{a}$, then $a$ is an accumulation point of $f(t) / t$ in $+\infty$.

Proof. Consider $f \in \mathcal{F}_{a}$ satisfying the condition

$$
\forall_{T>0} \forall_{\delta>0} \exists_{t \geq T}\left|\frac{f(t)}{t}-a\right|<\delta .
$$


If we put $T:=n$ and $\delta:=1 / n$, then we have

$$
\forall_{n>0} \exists_{t \geq n}\left|\frac{f(t)}{t}-a\right|<\frac{1}{n}
$$

This means that $a$ is an accumulation point of $f(t) / t$ in $+\infty$.

Assume that $a$ is not an accumulation point of $f(t) / t$ in $+\infty$. This implies that

$$
\exists_{T>0} \exists_{\delta>0} \forall_{t \geq T}\left|\frac{f(t)}{t}-a\right| \geq \delta
$$

Hence

$$
\mathrm{I}(f, a) \geq \int_{T}^{+\infty} \frac{|f(t)+a t|}{1+t^{2}} \mathrm{~d} t \geq \int_{T}^{+\infty} \frac{\delta t}{1+t^{2}} \mathrm{~d} t
$$

Since the last integral is divergent, this contradicts the assumption $f \in \mathcal{F}_{a}$, which completes the proof.

Proposition 2.8. If $f \in \mathcal{F}_{a}$, then a is an accumulation point of $f(t) / t$ in $-\infty$.

Proof. Consider $\tilde{f}(t):=-f(-t)$. By Proposition 2.4 we have $\tilde{f} \in \mathcal{F}_{a}$ and by Proposition 2.7 we obtain that $a$ is an accumulation point of $\tilde{f}(t) / t$ in $+\infty$. This is equivalent to that $a$ is an accumulation point of $f(t) / t$ in $-\infty$ and completes the proof.

Theorem 2.9. If $f \in \mathcal{F}_{a}$, then $\lim _{t \rightarrow+\infty} f(t) / t=a$.

Proof. Note, that by Proposition $2.7 a$ is the accumulation point of $f(t) / t$ in $+\infty$. Assume that there exists $b \in \mathbb{R}, b \neq a$ which is an accumulation point of $f(t) / t$ in $+\infty$, i.e. there exists a sequence $\left\{t_{n}\right\}, t_{n}>0, t_{n} \rightarrow+\infty$, such that

$$
\forall_{\varepsilon>0} \exists_{\tilde{n}} \forall_{n \geq \tilde{n}}\left|\frac{f\left(t_{n}\right)}{t_{n}}-b\right|<\varepsilon .
$$

Set $\varepsilon:=|a-b| / 3$ and denote

$$
s_{n}:=\frac{2 b+a}{2 a+b} t_{n}
$$

In view of Proposition 2.5 we may restrict our consideration to $a \geq 0$ and $b \geq 0$.

If $b>a \geq 0$, then $s_{n}>t_{n}$ and for $t \in\left[t_{n}, s_{n}\right]$ we have the following estimate

$$
f(t)-a t \geq f\left(t_{n}\right)-a s_{n}>\left(b-\epsilon-a \frac{2 b+a}{2 a+b}\right) t_{n}=\frac{(b-a)(2 b+a)}{3(2 a+b)} t_{n}>0 .
$$

We chose from $\left\{t_{n}\right\}$ a subsequence $\left\{t_{n_{k}}\right\}, k=1,2,3, \ldots$ such that $t_{n_{1}}=t_{\tilde{n}}$ and for all $k$ holds

$$
t_{n_{k+1}}>s_{n_{k}}
$$


Hence, for $t \in\left[t_{n}, s_{n}\right]$ we have

$$
\begin{aligned}
\mathrm{I}(f, a) & \geq \int_{0}^{+\infty} \frac{|f(t)-a t|}{1+t^{2}} \mathrm{~d} t=\int_{0}^{t_{n_{1}}} \frac{|f(t)-a t|}{1+t^{2}} \mathrm{~d} t+\int_{t_{n_{1}}}^{+\infty} \frac{|f(t)-a t|}{1+t^{2}} \mathrm{~d} t \\
& \geq \sum_{n=1}^{+\infty} \int_{t_{n_{k}}}^{t_{n_{k+1}}} \frac{|f(t)-a t|}{1+t^{2}} \mathrm{~d} t \geq \sum_{n=1}^{+\infty} \int_{t_{n_{k}}}^{s_{n_{k}}} \frac{|f(t)-a t|}{1+t^{2}} \mathrm{~d} t \\
& \geq \sum_{n=1}^{+\infty} \int_{t_{n_{k}}}^{s_{n_{k}}} \frac{(b-a)(2 b+a) t_{n_{k}}}{3(2 a+b)\left(1+t_{n_{k}}^{2}\right)} \mathrm{d} t \\
& =\sum_{n=1}^{+\infty} \frac{(b-a)(2 b+a)\left(s_{n_{k}}-t_{n_{k}}\right) t_{n_{k}}}{3(2 a+b)\left(1+s_{n_{k}}^{2}\right)} \\
& =\sum_{n=1}^{+\infty} \frac{(b-a)^{2}(2 b+a) t_{n_{k}}^{2}}{3\left[(2 a+b)^{2}+(2 b+a)^{2} t_{n_{k}}^{2}\right]} .
\end{aligned}
$$

Observe, that

$$
\lim _{n \rightarrow+\infty} \frac{(b-a)^{2}(2 b+a) t_{n_{k}}^{2}}{3\left[(2 a+b)^{2}+(2 b+a)^{2} t_{n_{k}}^{2}\right]}=\frac{(b-a)^{2}}{3(2 b+a)} \neq 0 .
$$

If $a>b \geq 0$, then $s_{n}<t_{n}$ and for $t \in\left[s_{n}, t_{n}\right]$ we have the following estimate

$$
f(t)-a t \leq f\left(t_{n}\right)-a s_{n}<\left(b+\epsilon-a \frac{2 b+a}{2 a+b}\right) t_{n}=\frac{(b-a)(2 b+a)}{3(2 a+b)} t_{n}<0 .
$$

We chose from $\left\{s_{n}\right\}$ a subsequence $\left\{s_{n_{k}}\right\}, k=1,2,3, \ldots$ such that $s_{n_{1}}=s_{\tilde{n}}$ and for all $k$ holds

$$
s_{n_{k+1}}>t_{n_{k}}
$$

Hence, for $t \in\left[s_{n}, t_{n}\right]$ we have

$$
\begin{aligned}
\mathrm{I}(f, a) & \geq \int_{0}^{+\infty} \frac{|f(t)-a t|}{1+t^{2}} \mathrm{~d} t=\int_{0}^{s_{n_{1}}} \frac{|f(t)-a t|}{1+t^{2}} \mathrm{~d} t+\int_{s_{n_{1}}}^{+\infty} \frac{|f(t)-a t|}{1+t^{2}} \mathrm{~d} t \\
& \geq \sum_{n=1}^{+\infty} \int_{s_{n_{k}}}^{s_{n_{k+1}}} \frac{|f(t)-a t|}{1+t^{2}} \mathrm{~d} t \geq \sum_{n=1}^{+\infty} \int_{s_{n_{k}}}^{t_{n_{k}}} \frac{|f(t)-a t|}{1+t^{2}} \mathrm{~d} t \\
& \geq \sum_{n=1}^{+\infty} \int_{s_{n_{k}}}^{t_{n_{k}}} \frac{(a-b)(2 b+a) t_{n_{k}}}{3(2 a+b)\left(1+t_{n_{k}}^{2}\right)} \mathrm{d} t=\sum_{n=1}^{+\infty} \frac{(a-b)(2 b+a)\left(t_{n_{k}}-s_{n_{k}}\right) t_{n_{k}}}{3(2 a+b)\left(1+t_{n_{k}}^{2}\right)} \\
& =\sum_{n=1}^{+\infty} \frac{(a-b)^{2}(2 b+a) t_{n_{k}}^{2}}{3(2 a+b)^{2}\left(1+t_{n_{k}}^{2}\right)} .
\end{aligned}
$$


Observe, that

$$
\lim _{n \rightarrow+\infty} \frac{(a-b)^{2}(2 b+a) t_{n_{k}}^{2}}{3(2 a+b)^{2}\left(1+t_{n_{k}}^{2}\right)}=\frac{(a-b)^{2}(2 b+a)}{3(2 a+b)^{2}} \neq 0 .
$$

Finally, (2.1) and (2.2), together, imply that $\mathrm{I}(f, a)=+\infty$, which contradicts the assumption $f \in \mathcal{F}$. Hence

$$
\lim _{t \rightarrow+\infty} f(t) / t=a,
$$

which completes the proof.

Theorem 2.10. If $f \in \mathcal{F}_{a}$, then $\lim _{t \rightarrow-\infty} f(t) / t=a$.

Proof. Consider $\tilde{f}(t):=-f(-t)$. By Proposition 2.4 we have $\tilde{f} \in \mathcal{F}_{a}$ and by Theorem 2.9 we obtain

$$
\lim _{t \rightarrow+\infty} \tilde{f}(t) / t=a
$$

This is equivalent to

$$
\lim _{t \rightarrow-\infty} f(t) / t=a
$$

and completes the proof.

Remark 2.11. Every function $f \in \mathcal{F}_{a}$ has the form

$$
\mathbb{R} \ni t \mapsto f(t)=a t+g(t),
$$

where $g(t) / t \rightarrow 0$ as $|t| \rightarrow+\infty$.

3. The harmonic extension $\boldsymbol{H}[\boldsymbol{f}]$. We introduce a harmonic extension of $f \in \mathcal{F}$ from $\mathbb{R}$ to $\mathbb{C}^{+}$. By the definition of the class $\mathcal{F}$ the following definition makes sense.

Definition 3.1. For $f \in \mathcal{F}_{a}$ we define $H[f]: \mathbb{C}^{+} \rightarrow \mathbb{C}^{+}$as follows

$$
H[f](z):=a z+P[g](z),
$$

where $g$ is related to $f$ by $(2.3)$ and

$$
P[g](z):=\int_{-\infty}^{+\infty} P_{z}(t) g(t) \mathrm{d} t
$$

is the Poisson integral for $\mathbb{C}^{+}$and

$$
P_{z}(t):=\frac{1}{\pi} \frac{\operatorname{Im}\{z\}}{|z-t|^{2}}
$$

is the Poisson kernel for $\mathbb{C}^{+}$. 
Note, that $P[g](z) \in \mathbb{R}$ for every $z \in \mathbb{C}^{+}$and let us denote

$$
U(z):=\operatorname{Re}\{H[f](z)\}=a \operatorname{Re}\{z\}+P[g](z)
$$

and

$$
V(z):=\operatorname{Im}\{H[f](z)\}=a \operatorname{Im}\{z\} .
$$

Throughout this paper $U$ and $V$ will always mean $\operatorname{Re}\{H[f]\}$ and $\operatorname{Im}\{H[f]\}$, respectively.

Recall that the biLipschitz condition on $f$, i.e.

$$
\exists_{L_{1}, L_{2}>0} \forall_{t_{1}, t_{2} \in \mathbb{R}} L_{2}\left|t_{2}-t_{1}\right| \leq\left|f\left(t_{2}\right)-f\left(t_{1}\right)\right| \leq L_{1}\left|t_{2}-t_{1}\right|
$$

is the necessary condition for $H[f]$ to be quasiconformal (see [2]).

Proposition 3.2. If $f \in \mathcal{F}_{a}$ satisfies the biLipschitz condition (3.2), then $H[f]$ is a homeomorphism of $\mathbb{C}^{+}$onto itself.

Proof. Fix $y>0$ and let $z_{1}=x_{1}+i y, z_{2}=x_{2}+i y$, where $x_{1}, x_{2} \in \mathbb{R}$. Since $P_{z}(t)>0, t \in \mathbb{R}$ and

$$
\int_{-\infty}^{+\infty} P_{z}(t) \mathrm{d} t=1, \quad z \in \mathbb{C}^{+}
$$

we can write

$$
\begin{aligned}
U\left(z_{1}\right)- & U\left(z_{2}\right)=a x_{1}+P[g]\left(z_{1}\right)-a x_{2}-P[g]\left(z_{2}\right) \\
= & \int_{-\infty}^{+\infty} \frac{1}{\pi} \frac{y}{\left(x_{1}-t\right)^{2}+y^{2}}\left[a x_{1}+g(t)\right] \mathrm{d} t \\
& -\int_{-\infty}^{+\infty} \frac{1}{\pi} \frac{y}{\left(x_{2}-t\right)^{2}+y^{2}}\left[a x_{2}+g(t)\right] \mathrm{d} t \\
= & \int_{-\infty}^{+\infty} \frac{1}{\pi} \frac{y}{s^{2}+y^{2}}\left[a\left(x_{1}-s\right)+g\left(x_{1}-s\right)-a\left(x_{2}-s\right)-g\left(x_{2}-s\right)\right] \mathrm{d} s \\
= & \int_{-\infty}^{+\infty} \frac{1}{\pi} \frac{y}{s^{2}+y^{2}}\left[f\left(x_{1}-s\right)-f\left(x_{2}-s\right)\right] \mathrm{d} s .
\end{aligned}
$$

Because $f$ increases, then $U\left(z_{1}\right)>U\left(z_{2}\right)$ for $x_{1}>x_{2}$. Hence $U$ is univalent on every horizontal line. Since $V(z)=a \operatorname{Im}\{z\}, H[f]$ is univalent.

To show that $U$ maps every horizontal line in the upper halfplane onto $\mathbb{R}$, we fix $y>0$ and observe that

$$
U(x+i y)-U(i y)=\int_{-\infty}^{+\infty} \frac{1}{\pi} \frac{y}{s^{2}+y^{2}}[f(x-s)-f(-s)] \mathrm{d} s .
$$

Let $x>0$. Since $f$ increases and by applying the biLipschitz condition (3.2), we have

$$
U(x+i y)-U(i y) \geq \int_{-\infty}^{+\infty} \frac{1}{\pi} \frac{y}{s^{2}+y^{2}} L_{2}|x| \mathrm{d} s=L_{2} x .
$$


Let $x<0$. Analogically we obtain

$$
U(x+i y)-U(i y) \leq-\int_{-\infty}^{+\infty} \frac{1}{\pi} \frac{y}{s^{2}+y^{2}} L_{2}|x| \mathrm{d} s=L_{2} x .
$$

Since $V(z)=a \operatorname{Im}\{z\}, H[f]\left(\mathbb{C}^{+}\right)=\mathbb{C}^{+}$.

The following example shows that not every function $f \in \mathcal{F}$ has the extension $H[f]$ which is quasiconformal.

Example 3.3. Consider the function $f: \mathbb{R} \rightarrow \mathbb{R}$ defined as $f(t)=t+$ $|t|^{1 / 2} \operatorname{sgn} t$. Obviously, $f \in \mathcal{F}_{1} \subset \mathcal{F}$ since

$$
\int_{-\infty}^{+\infty} \frac{|t|^{1 / 2}}{1+t^{2}} \mathrm{~d} t<+\infty
$$

On the other hand, we have

$$
\left|f\left(t_{1}\right)-f\left(t_{2}\right)\right|=\left|t_{1}-t_{2}\right|\left(1+\frac{1}{\sqrt{t_{1}}+\sqrt{t_{2}}}\right),
$$

where $t_{1}, t_{2}>0$. Hence, we see that

$$
\forall_{L>0} \exists_{t_{1}, t_{2}>0} 1+\frac{1}{\sqrt{t_{1}}+\sqrt{t_{2}}}>L
$$

e.g. putting $t_{2}:=t_{1} / 4:=1 /\left(9 L^{2}\right)$. This means that $f$ is not biLipschitz and so it cannot have quasiconformal extension to the upper halfplane.

4. Estimates of partial derivatives of $\boldsymbol{H}[\boldsymbol{f}]$. Let $f \in \mathcal{F}_{a}$ and $z=x+i y$. We compute partial derivatives of $U$ and $V$.

$$
\begin{aligned}
\frac{\partial U}{\partial x}(z) & =a+\frac{\partial}{\partial x}(P[g](z))=a+\int_{-\infty}^{+\infty} \frac{1}{\pi} \frac{-2 y(x-t)}{\left[(x-t)^{2}+y^{2}\right]^{2}} g(t) \mathrm{d} t \\
& =a+\int_{0}^{+\infty} \frac{1}{\pi} \frac{2 y s}{\left(s^{2}+y^{2}\right)^{2}}[g(x+s)-g(x-s)] \mathrm{d} s \\
\frac{\partial U}{\partial y}(z) & =\frac{\partial}{\partial y}(P[g](z))=\int_{-\infty}^{+\infty} \frac{1}{\pi} \frac{(x-t)^{2}-y^{2}}{\left[(x-t)^{2}+y^{2}\right]^{2}} g(t) \mathrm{d} t \\
& =\int_{0}^{+\infty} \frac{1}{\pi} \frac{s^{2}-y^{2}}{\left(s^{2}+y^{2}\right)^{2}}[g(x+s)+g(x-s)] \mathrm{d} s, \\
\frac{\partial V}{\partial x}(z) & =0 \\
\frac{\partial V}{\partial y}(z) & =a .
\end{aligned}
$$

First, we give the estimates on $\partial U / \partial x$ under assumption, that $f \in \mathcal{F}$ is biLipschitz only. 
Theorem 4.1. If $f \in \mathcal{F}_{a}$ satisfies the biLipschitz condition (3.2), then

$$
L_{2} \leq \frac{\partial U}{\partial x}(z) \leq L_{1}, \quad z \in \mathbb{C}^{+} .
$$

Proof. Observe, that (3.2) implies

$$
2\left(L_{2}-a\right) s \leq g(x+s)-g(x-s) \leq 2\left(L_{1}-a\right) s
$$

for every $s>0$. Let $z=x+i y$. Then

$$
\begin{aligned}
\frac{\partial U}{\partial x}(z) & =a+\int_{0}^{+\infty} \frac{1}{\pi} \frac{2 y s}{\left(s^{2}+y^{2}\right)^{2}}[g(x+s)-g(x-s)] \mathrm{d} s \\
& \leq a+\int_{0}^{+\infty} \frac{1}{\pi} \frac{4 y s^{2}}{\left(s^{2}+y^{2}\right)^{2}}\left(L_{1}-a\right) \mathrm{d} s=L_{1}, \\
\frac{\partial U}{\partial x}(z) & =a+\int_{0}^{+\infty} \frac{1}{\pi} \frac{2 y s}{\left(s^{2}+y^{2}\right)^{2}}[g(x+s)-g(x-s)] \mathrm{d} s \\
& \geq a+\int_{0}^{+\infty} \frac{1}{\pi} \frac{4 y s^{2}}{\left(s^{2}+y^{2}\right)^{2}}\left(L_{2}-a\right) \mathrm{d} s=L_{2} .
\end{aligned}
$$

As a corollary from the estimates of $\partial U / \partial x$ we obtain the estimates of the Jacobian $\mathrm{J}_{H[f]}$ of $H[f]$ defined in (1.1).

Corollary 4.2. If $f \in \mathcal{F}_{a}$ satisfies the biLipschitz condition (3.2), then

$$
a L_{2} \leq \mathrm{J}_{H[f]}(z) \leq a L_{1}, \quad z \in \mathbb{C}^{+} .
$$

Proof. We can rewrite the Jacobian of $H[f]$ in the form

$$
J_{H[f]}=\frac{\partial U}{\partial x} \frac{\partial V}{\partial y}-\frac{\partial U}{\partial y} \frac{\partial V}{\partial x} .
$$

Since $\partial V / \partial x=0$ and $\partial V / \partial y=a$, by applying the inequalities (4.2) the proof is completed.

Now, we give the estimate of $\partial U / \partial y$ under an additional assumption on $f$, but first we formulate the following lemma.

Lemma 4.3. If $f \in \mathcal{F}$ is absolutely continuous function, then

$$
\frac{\partial U}{\partial y}(z)=\int_{0}^{+\infty} \frac{1}{\pi} \frac{s}{s^{2}+y^{2}}\left[f^{\prime}(x+s)-f^{\prime}(x-s)\right] \mathrm{d} s .
$$

Proof. Recall that

$$
\frac{\partial U}{\partial y}(z)=\int_{0}^{+\infty} \frac{1}{\pi} \frac{s^{2}-y^{2}}{\left(s^{2}+y^{2}\right)^{2}}[g(x+s)+g(x-s)] \mathrm{d} s,
$$

where $z=x+i y$. Since $f$ is absolutely continuous, $f^{\prime}$ exists almost everywhere and for almost all $t_{1}, t_{2} \in \mathbb{R}$

$$
f^{\prime}\left(t_{1}\right)-f^{\prime}\left(t_{2}\right)=g^{\prime}\left(t_{1}\right)-g^{\prime}\left(t_{2}\right) .
$$


Hence, integrating by parts we have

$$
\begin{aligned}
\frac{\partial U}{\partial y}(z)= & -\left.\frac{1}{\pi} \frac{s}{s^{2}+y^{2}}[g(x+s)+g(x-s)]\right|_{0} ^{+\infty} \\
& +\int_{0}^{+\infty} \frac{1}{\pi} \frac{s}{s^{2}+y^{2}}\left[g^{\prime}(x+s)-g^{\prime}(x-s)\right] \mathrm{d} s .
\end{aligned}
$$

Since, by Theorem 2.9,

$$
\lim _{t \rightarrow+\infty} \frac{g(t)}{t}=0
$$

the proof is completed.

Recall, that a continuous function $\varphi$ is said to be Dini-continuous with respect to spherical distance if it satisfies the following condition

$$
\int_{0}^{\varsigma} \frac{\omega(t)}{t} \mathrm{~d} t=M_{\varsigma}<+\infty
$$

for some $\varsigma \in(0,1]$, where $\omega:[0,1] \rightarrow[0,1]$,

$$
\omega(t):=\sup \left\{d_{s}\left(\varphi\left(t_{1}\right), \varphi\left(t_{2}\right)\right): d_{s}\left(t_{1}, t_{2}\right)<t\right\}
$$

is the modulus of continuity of $\varphi$ with respect to spherical distance $d_{s}$,

$$
d_{s}\left(t_{1}, t_{2}\right):=\frac{\left|t_{1}-t_{2}\right|}{\sqrt{1+t_{1}^{2}} \sqrt{1+t_{2}^{2}}} .
$$

Obviously, $\omega$ is non-decreasing function and

$$
d_{s}\left(\varphi\left(t_{1}\right), \varphi\left(t_{2}\right)\right) \leq \omega\left(d_{s}\left(t_{1}, t_{2}\right)\right)
$$

holds for all $t_{1}, t_{2} \in \mathbb{R}$.

Remark 4.4. If $f$ satisfies the biLipschitz condition (3.2) and $f^{\prime}$ is Dinicontinuous with respect to spherical distance a.e. in $\mathbb{R}$, then $f^{\prime}$ exists everywhere in $\hat{\mathbb{R}}:=\mathbb{R} \cup\{\infty\}$ and $L_{2} \leq\left|f^{\prime}(t)\right| \leq L_{1}, t \in \hat{\mathbb{R}}$. In particular, there exists finite value of $f^{\prime}$ at the point $\infty$. If, additionally, $f \in \mathcal{F}_{a}$, then by Remark $2.11 f$ is of the form (2.3) and so we have

$$
\lim _{t \rightarrow+\infty} f^{\prime}(t)=\lim _{t \rightarrow-\infty} f^{\prime}(t)=a .
$$

Theorem 4.5. If $f \in \mathcal{F}$ satisfies the biLipschitz condition (3.2) and if $f^{\prime}$ is Dini-continuous with respect to spherical distance (4.3), then

$$
\left|\frac{\partial U}{\partial y}(z)\right| \leq \frac{2\left(1+L_{1}^{2}\right)}{\pi}\left[\frac{M_{\varsigma}}{\sqrt{1-\delta^{2}}}+\log \left(\frac{1+\sqrt{1-\delta^{2}}}{\delta}\right)\right],
$$

where $\delta:=\min \left\{\varsigma, 1 / \sqrt{1+M_{\varsigma}}\right\}$ and $\varsigma, M_{\varsigma}$ satisfy (4.3). 
Proof. Since $f$ is biLipschitz, $f$ is absolutely continuous and by Lemma 4.3 we have

$$
\begin{aligned}
\left|\frac{\partial U}{\partial y}(z)\right| & =\left|\frac{1}{\pi} \int_{0}^{+\infty} \frac{s}{s^{2}+y^{2}}\left[g^{\prime}(x+s)-g^{\prime}(x-s)\right] \mathrm{d} s\right| \\
& \leq \frac{1}{\pi} \int_{0}^{+\infty} \frac{\left|g^{\prime}(x+s)-g^{\prime}(x-s)\right|}{s} \mathrm{~d} s .
\end{aligned}
$$

From the Dini-continuity condition with respect to spherical distance (4.3) we have that (4.4) holds for $f^{\prime}$ and so we obtain

$$
\begin{aligned}
\left|\frac{\partial U}{\partial y}(z)\right| \leq \frac{1}{\pi} \int_{0}^{+\infty} & {\left[\frac{\sqrt{1+\left[f^{\prime}(x+s)\right]^{2}} \sqrt{1+\left[f^{\prime}(x-s)\right]^{2}}}{s}\right.} \\
& \left.\times \omega\left(\frac{2 s}{\sqrt{1+(x+s)^{2}} \sqrt{1+(x-s)^{2}}}\right)\right] \mathrm{d} s .
\end{aligned}
$$

Again, the biLipschitz condition for $f$ gives

$$
\left|\frac{\partial U}{\partial y}(z)\right| \leq \frac{\left(1+L_{1}^{2}\right)}{\pi} \int_{0}^{+\infty} \frac{1}{s} \omega\left(\frac{2 s}{\sqrt{1+(x+s)^{2}} \sqrt{1+(x-s)^{2}}}\right) \mathrm{d} s .
$$

Setting

$$
t:=\frac{2 s}{\sqrt{1+(x+s)^{2}} \sqrt{1+(x-s)^{2}}},
$$

we have

$$
t^{\prime}=\frac{-2 s^{4}+2\left(1+x^{2}\right)^{2}}{\left(\sqrt{1+(x+s)^{2}} \sqrt{1+(x-s)^{2}}\right)^{3}}=\frac{t^{3}\left[-2 s^{4}+2\left(1+x^{2}\right)^{2}\right]}{4 s^{3}} .
$$

Let

$$
\begin{aligned}
& A:=t^{2}, \quad B:=\left[2 t^{2}\left(1-x^{2}\right)-4\right], \quad C:=t^{2}\left(1+x^{2}\right)^{2}, \\
& \Delta:=B^{2}-4 A C=16\left(1-t^{2}\right)\left(1+x^{2} t^{2}\right) .
\end{aligned}
$$

To apply the substitution (4.6) to the last integral we need to divide it into two integrals from 0 to $\sqrt{1+x^{2}}$ and from $\sqrt{1+x^{2}}$ to $+\infty$. Then we obtain

$$
\begin{aligned}
& \left|\frac{\partial U}{\partial y}(z)\right| \leq \frac{4\left(1+L_{1}^{2}\right)}{\pi} \int_{0}^{+\infty} \frac{s^{2}}{\left(B s^{2}+2 C\right)} \frac{\omega(t)}{t^{3}} t^{\prime} \mathrm{d} s \\
& =\frac{4\left(1+L_{1}^{2}\right)}{\pi}\left[\int_{0}^{\sqrt{1+x^{2}}} \frac{1}{\left(B+\frac{2 C}{s^{2}}\right)} \frac{\omega(t)}{t^{3}} t^{\prime} \mathrm{d} s+\int_{\sqrt{1+x^{2}}}^{+\infty} \frac{1}{\left(B+\frac{2 C}{s^{2}}\right)} \frac{\omega(t)}{t^{3}} t^{\prime} \mathrm{d} s\right] .
\end{aligned}
$$

From (4.6) we compute two solutions

$$
s^{2}=\frac{-B-\sqrt{\Delta}}{2 A} \text { and } s^{2}=\frac{-B+\sqrt{\Delta}}{2 A}
$$


for $t \in(0,1)$. Hence, we have

$$
\begin{aligned}
\left|\frac{\partial U}{\partial y}(z)\right| & \leq \frac{4\left(1+L_{1}^{2}\right)}{\pi} \int_{0}^{1} \frac{1}{\sqrt{\Delta}} \frac{\omega(t)}{t} \mathrm{~d} t+\frac{4\left(1+L_{1}^{2}\right)}{\pi} \int_{1}^{0} \frac{-1}{\sqrt{\Delta}} \frac{\omega(t)}{t} \mathrm{~d} t \\
& =\frac{8\left(1+L_{1}^{2}\right)}{\pi} \int_{0}^{1} \frac{1}{\sqrt{\Delta}} \frac{\omega(t)}{t} \mathrm{~d} t \leq \frac{2\left(1+L_{1}^{2}\right)}{\pi} \int_{0}^{1} \frac{1}{\sqrt{1-t^{2}}} \frac{\omega(t)}{t} \mathrm{~d} t .
\end{aligned}
$$

Since, by definition, $\omega(t) \leq 1$ and $\omega$ satisfies (4.3),

$$
\begin{aligned}
\left|\frac{\partial U}{\partial y}(z)\right| & \leq \frac{2\left(1+L_{1}^{2}\right)}{\pi}\left[\int_{0}^{\delta} \frac{1}{\sqrt{1-t^{2}}} \frac{\omega(t)}{t} \mathrm{~d} t+\int_{\delta}^{1} \frac{\omega}{t \sqrt{1-t^{2}}} \mathrm{~d} t\right] \\
& \leq \frac{2\left(1+L_{1}^{2}\right)}{\pi} \begin{cases}\frac{1}{\sqrt{1-\varsigma^{2}}} \int_{0}^{\varsigma} \frac{\omega(t)}{t} \mathrm{~d} t+\int_{\varsigma}^{1} \frac{1}{t \sqrt{1-t^{2}}} \mathrm{~d} t, \quad \delta \geq \varsigma, \\
\frac{1}{\sqrt{1-\delta^{2}}} \int_{0}^{\delta} \frac{\omega(t)}{t} \mathrm{~d} t+\int_{\delta}^{1} \frac{1}{t \sqrt{1-t^{2}}} \mathrm{~d} t, \quad \delta<\varsigma\end{cases} \\
& \leq \frac{2\left(1+L_{1}^{2}\right)}{\pi} \begin{cases}\frac{M_{\varsigma}}{\sqrt{1-\varsigma^{2}}}+\log \left(\frac{1+\sqrt{1-\varsigma^{2}}}{\varsigma}\right), & \delta \geq \varsigma, \\
\frac{M_{\varsigma}}{\sqrt{1-\delta^{2}}}+\log \left(\frac{1+\sqrt{1-\delta^{2}}}{\delta}\right), & \delta<\varsigma .\end{cases}
\end{aligned}
$$

Simple calculation shows that the above estimate is the best when $\delta=$ $\min \left\{\varsigma, 1 / \sqrt{1+M_{\varsigma}}\right\}$ and the proof is completed.

In particular, if $\varphi$ is Hölder-continuous with respect to spherical distance $d_{s}$, i.e.

$$
d_{s}\left(\varphi\left(t_{1}\right), \varphi\left(t_{2}\right)\right) \leq \lambda d_{s}\left(t_{1}, t_{2}\right)^{\alpha}
$$

for all $t_{1}, t_{2} \in \mathbb{R}$ and some constants $\lambda>0$ and $\alpha \in(0,1]$, then $\varphi$ is also Dini-continuous with respect to spherical distance.

We have the following corollary from the proof of Theorem 4.5.

Corollary 4.6. If $f \in \mathcal{F}$ satisfies the biLipschitz condition (3.2) and $f^{\prime}$ is Hölder-continuous with respect to spherical distance (4.7), then

$$
\left|\frac{\partial U}{\partial y}(z)\right| \leq \frac{\lambda\left(1+L_{1}^{2}\right)}{\pi}\left\{\begin{array}{cc}
\mathrm{B}\left(\frac{\alpha}{2}, \frac{1}{2} ; 1\right), & \lambda \leq 1 \\
\mathrm{~B}\left(\frac{\alpha}{2}, \frac{1}{2} ; \lambda^{-1 / \alpha}\right) & \\
+\frac{2}{\lambda} \log \left(\lambda^{1 / \alpha}+\sqrt{\lambda^{2 / \alpha}-1}\right), & \lambda>1 .
\end{array}\right.
$$

where B denotes the incomplete beta function and $\lambda, \alpha$ satisfy (4.7).

Proof. From the proof of Theorem 4.5 we have

$$
\left|\frac{\partial U}{\partial y}(z)\right| \leq \frac{2\left(1+L_{1}^{2}\right)}{\pi} \int_{0}^{1} \frac{\omega(t)}{t \sqrt{1-t^{2}}} \mathrm{~d} t
$$

where $\omega$ is the modulus of continuity of $f^{\prime}$ with respect to spherical distance. Since $f^{\prime}$ satisfies $(4.7)$ and $\omega(t) \leq 1$, we have

$$
\omega(t) \leq \min \left\{1, \lambda t^{\alpha}\right\} .
$$


Hence

$$
\left|\frac{\partial U}{\partial y}(z)\right| \leq \frac{2\left(1+L_{1}^{2}\right)}{\pi} \begin{cases}\int_{0}^{1} \frac{\lambda t^{\alpha}}{t \sqrt{1-t^{2}}} \mathrm{~d} t, & \lambda \leq 1, \\ \int_{0}^{\lambda^{-1 / \alpha}} \frac{\lambda t^{\alpha}}{t \sqrt{1-t^{2}}} \mathrm{~d} t+\int_{\lambda^{-1 / \alpha}}^{1} \frac{1}{t \sqrt{1-t^{2}}} \mathrm{~d} t, & \lambda>1 .\end{cases}
$$

Finally, recall that for $a>0, b>0$ and $c \in[0,1]$ the incomplete beta function is defined by the formula (see [4])

$$
\mathrm{B}(a, b ; c):=\int_{0}^{c} t^{a-1}(1-t)^{b-1} \mathrm{~d} t .
$$

Hence, the proof is completed.

5. Quasiconformality of $\boldsymbol{H}[\boldsymbol{f}]$. Using estimates on partial derivatives of the extension $H[f]$ we are able to estimate its maximal dilatation $\mathrm{D}_{H[f]}$, which is the main tool in studying quasiconformality of $H[f]$.

Theorem 5.1. If $f \in \mathcal{F}_{a}$ satisfies the biLipschitz condition (3.2) and $|\partial U / \partial y| \leq A$ for some $A>0$, then

$$
\mathrm{D}_{H[f]}(z) \leq \frac{L_{1}}{a}+\frac{A^{2}+a^{2}}{a L_{2}}, \quad z \in \mathbb{C}^{+} .
$$

Proof. We have

$$
\begin{aligned}
\mathrm{D}_{H[f]}(z) & \leq 2 \frac{|\partial H(z)|^{2}+|\bar{\partial} H(z)|^{2}}{\mathrm{~J}_{H[f]}(z)} \\
& =\frac{\left(\frac{\partial U}{\partial x}(z)\right)^{2}+\left(\frac{\partial U}{\partial y}(z)\right)^{2}+\left(\frac{\partial V}{\partial x}(z)\right)^{2}+\left(\frac{\partial V}{\partial y}(z)\right)^{2}}{\frac{\partial U}{\partial x}(z) \frac{\partial V}{\partial y}(z)-\frac{\partial U}{\partial y}(z) \frac{\partial V}{\partial x}(z)} .
\end{aligned}
$$

Combining this with (4.1) we obtain

$$
\mathrm{D}_{H[f]}(z) \leq \frac{\frac{\partial U}{\partial x}(z)}{a}+\frac{\left(\frac{\partial U}{\partial y}(z)\right)^{2}+a^{2}}{a \frac{\partial U}{\partial x}(z)} .
$$

Applying (4.2) and the assumption $|\partial U / \partial y| \leq A$ the theorem follows.

Theorem 5.2. If $f \in \mathcal{F}_{a}$ satisfies the biLipschitz condition (3.2) and if $f^{\prime}$ is Dini-continuous with respect to spherical distance (4.3), then

$$
\mathrm{D}_{H[f]}(z) \leq \frac{L_{1}}{a}+\frac{\frac{4}{\pi^{2}}\left(1+L_{1}^{2}\right)^{2}\left[\frac{M_{\varsigma}}{\sqrt{1-\delta^{2}}}+\log \left(\frac{1+\sqrt{1-\delta^{2}}}{\delta}\right)\right]^{2}+a^{2}}{a L_{2}}, \quad z \in \mathbb{C}^{+},
$$

where $\delta:=\min \left\{\varsigma, 1 / \sqrt{1+M_{\varsigma}}\right\}$ and $\varsigma, M_{\varsigma}$ satisfy $(4.3)$.

Proof. Theorem 4.5 gives the estimate (4.5) on $|\partial U / \partial y|$. Hence, the theorem follows from Theorem 5.1. 
Theorem 5.3. If $f \in \mathcal{F}_{a}$ satisfies the biLipschitz condition (3.2) and $f^{\prime}$ is Hölder-continuous with respect to spherical distance (4.7), then

$$
\mathrm{D}_{H[f]}(z) \leq \frac{L_{1}}{a}+\frac{A^{2}+a^{2}}{a L_{2}}, \quad z \in \mathbb{C}^{+},
$$

where

$$
A=\frac{\lambda\left(1+L_{1}^{2}\right)}{\pi} \begin{cases}\mathrm{B}\left(\frac{\alpha}{2}, \frac{1}{2} ; 1\right), & \lambda \leq 1 \\ \mathrm{~B}\left(\frac{\alpha}{2}, \frac{1}{2} ; \lambda^{-1 / \alpha}\right)+\frac{2}{\lambda} \log \left(\lambda^{1 / \alpha}+\sqrt{\lambda^{2 / \alpha}-1}\right), & \lambda>1\end{cases}
$$

and $\mathrm{B}$ denotes the incomplete beta function and $\lambda, \alpha$ satisfy (4.7).

Proof. Corollary 4.6 gives the estimate (4.8) on $|\partial U / \partial y|$. Hence, the theorem follows from Theorem 5.1.

\section{REFERENCES}

[1] Ahlfors, L. V., Lectures on Quasiconformal Mappings, Van Nostrand Mathematical Studies, D. Van Nostrand, Princeton, 1966.

[2] Kalaj, D., Pavlović, M., Boundary correspondence under quasiconformal harmonic diffeomorphisms of a half-plane, Ann. Acad. Sci. Fenn. Ser. A. I. Math. 30 (2005), 159-165.

[3] Lehto, O., Virtanen, K. I., Quasiconformal Mappings in the Plane, 2nd ed., Grundlehren der matematischen Wissenschaften 126, Springer-Verlag, Berlin, 1973.

[4] Pearson, K., Tables of the Incomplete Beta-Function, Cambridge Univ. Press, Cambridge, 1934.

Andrzej Michalski

Department of Complex Analysis

Faculty of Mathematics and Natural Sciences

The John Paul II Catholic University of Lublin

ul. Konstantynów $1 \mathrm{H}$

20-950 Lublin, Poland

e-mail: amichal@kul.lublin.pl

Received September 10, 2007 